\title{
N-body/SPH study of dEs in a cluster environment
}

\author{
Dolf Michielsen, S. De Rijcke and H. Dejonghe \\ Sterrenkundig Observatorium, Ghent University, Krijgslaan 281, B-9000 Ghent, Belgium \\ email: dolf.michielsen@UGent.be
}

\begin{abstract}
Until recently, dwarf elliptical galaxies (dEs) were mostly believed to be gas-less systems, having lost their interstellar medium (ISM) through a galactic wind or through ram pressure stripping in the dense cluster environment. However, recent $\mathrm{H} \alpha$ and $\mathrm{H}$ I surveys indicate that some dEs in cluster environments are able to retain part of their gas (Drinkwater et al. (2001), Conselice et al. (2001)). In a detailed observational study of the ISM of a sample of Fornax dEs, we detected genuine dEs containing warm and cold gas (De Rijcke et al. (2003), Michielsen et al. (2004), Buyle et al. (2005)).

Using an N-body/SPH code, we explore the two gas-removing scenarios in order to assess their efficiency in removing the ISM from dEs. We simulated dEs subjected to ram pressure stripping by the intracluster medium (ICM) and found that, while small dEs are instantaneously stripped, more massive dEs are able to retain part of their ISM over long time-scales. Isolated dEs on the other hand are expected to lose their gas through a galactic wind. Our simulations show that supernova explosions can blow (super)bubbles in the ISM, thereby ejecting part, though not all, of the ISM.
\end{abstract}

Keywords. methods: n-body simulations, galaxies: dwarf, galaxies: ISM

\section{Ram pressure stripping}

We used a 3D SPH code to model ram pressure stripping from dEs that initially contain an ionized ISM in hydrostatic equilibrium with the Burkert dark matter (DM) potential (Burkert, 1995). For DM halo masses ranging from $10^{6}$ to $10^{10} \mathrm{M}_{\odot}$, two different sets of ISM initial density and pressure profiles were adopted. First the ISM is set up to be density confined within the ICM (referred to as DC), with a temperature proportional to the DM halo mass $\left(T_{\mathrm{ISM}} \propto M_{\mathrm{DM}}^{4 / 7}\right)$. This results in a one-parameter family of models (Mori \& Burkert, 2000). Second, the ISM is considered to be pressure confined within the surrounding ICM (referred to as PC), and we assume a fixed temperature $T_{\text {ISM }}=10^{4} \mathrm{~K}$. The PC conditions have much smaller ISM boundary radius than the DC conditions. The ICM properties chosen are typical for a moderate cluster like the Virgo Cluster $\left(T_{\mathrm{ICM}}=10^{7} \mathrm{~K}, n_{\mathrm{ICM}}=10^{-4} \mathrm{~cm}^{-3}\right)$, while the relative velocity of the galaxy through the cluster was fixed at $1000 \mathrm{~km} \mathrm{~s}^{-1}$.

When subjected to the ram pressure, the ISM of small dEs $\left(M_{\mathrm{DM}}<10^{8} \mathrm{M}_{\odot}\right)$ is instantaneously stripped. This was to be expected since the central thermal pressure of the ISM is lower than the ram pressure of the ICM. However, the central pressure in the more massive dwarfs $\left(M_{\mathrm{DM}} \geqslant 10^{9} \mathrm{M}_{\odot}\right)$ is considerably higher in the $\mathrm{PC}$, than in the $\mathrm{DC}$ simulations, due to the higher central densities. Therefore, these dEs are able to retain their ISM over several dynamical time-scales, during which the gas may cool and form stars. Star formation may be even enhanced by the increased density due to the ram pressure of the ICM. 


\section{Galactic winds}

We used a 3D N-body/SPH code including radiative cooling, star formation and feedback to model the formation of an isolated $\mathrm{dE}$. The $9 \times 10^{8} \mathrm{M}_{\odot} \mathrm{DM}$ halo is set up in a King distribution with concentration parameter $c=2$. Initially, the $10^{8} \mathrm{M}_{\odot}$ of gas is distributed homogeneously within the DM tidal radius. Star formation occurs in collapsing, cold and dense gas particles (Katz, 1992). Each newly formed star particle represents a simple stellar population. The most massive stars explode as SNe, each injecting $10^{50} \mathrm{ergs}$ of thermal energy into the surrounding gas.

Different tidal radii are adopted, giving different initial density distributions. The star formation rates (SFRs) differ significantly for models with different initial densities (Carraro et al., 2001). The densest model shows an intense starburst of 100 Myr, whereafter the remaining ISM is blown out in a galactic wind. Models with lower initial density have lower SFRs spanning longer time-scales. The SNe exploding in these low-density systems blow (super)bubbles in the ISM, and part of the hot, enriched gas escapes the galaxy, while part of the gas is able to fall back. The increased density caused by shocks enhances the star-formation. After an initial prolonged starburst phase, the low-density models keep on forming stars at a slow rate for several Gyr.

\section{Conclusions and outlook}

Our simulations show that the gas-removal processes in dEs are not always efficient. The ISM of more massive dEs can survive ram pressure stripping for long timescales, while galactic winds blow out only part of the ISM. These results corroborate our observations of genuine dEs containing a warm and cold ISM.

Until now, simulations of $\mathrm{dE}$ formation including star formation and feedback concentrated on isolated dEs. However, if ram pressure stripping is not instantaneous, the thermal pressure of a surrounding ICM could confine the galactic wind inside the $\mathrm{dE}$ so that more gas is available for star formation and enriched gas cannot easily escape the galaxy. Furthermore, the increased density induced by ram pressure is assumed to increase the SFR. We now have the tools to investigate this scenario. Simulating a variety of ICM and dE properties will shed more light on the formation and evolution of dEs in a dense environment.

\section{Acknowledgements}

D. M. acknowledges the financial support of the Bijzonder OnderzoeksFonds (Ghent University). S. D. R. acknowledges the financial support of the Belgian Fund for Scientific Research (F.W.O.).

\section{References}

Burkert, A. 1995, ApJ 447, 25

Buyle, P., De Rijcke, S., Michielsen, D., Baes, M. \& Dejonghe, H. 2005, MNRAS accepted (astro-ph/0504270)

Carraro, G., Chiosi C., Girardi, L. \& Lia, C., 2001, MNRAS 327, 69

Conselice, C.J., Gallagher, J.S. \& Wyse, R.F.G. 2003, AJ 125, 66

De Rijcke, S., Zeilinger, W.W., Dejonghe, H. \& Hau G.T.K. 2003b, MNRAS 339, 225

Drinkwater M.J., Gregg M.D., Holman B.A. \& Brown M.J.I. 2001, MNRAS 326, 1076

Katz, N. 1992, ApJ 391, 502

Michielsen, D., De Rijcke, S., Zeilinger, W.W., Prugniel, P., Dejonghe, H. \& Roberts, S. 2004, MNRAS 353, 1293

Mori, M. \& Burkert, A. 2000, ApJ 538, 339 